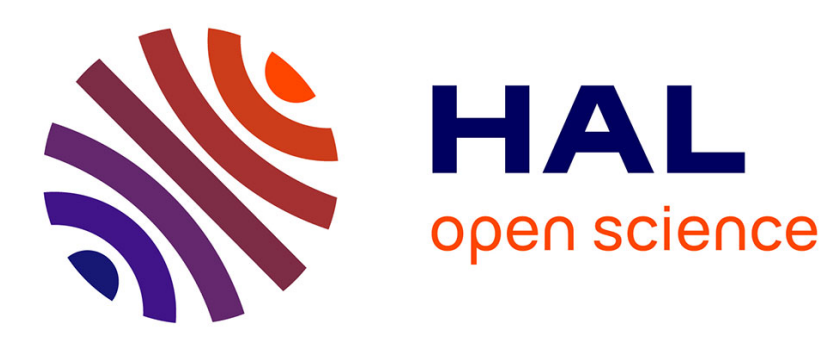

\title{
Obtention de la forme sexuée de Rhizoctonia solani Kühn : Thanatephorus cucumeris (Frank) Donk \\ Pierre Camporota
}

\section{To cite this version:}

Pierre Camporota. Obtention de la forme sexuée de Rhizoctonia solani Kühn: Thanatephorus cucumeris (Frank) Donk. Agronomie, 1986, 6 (3), pp.305-307. hal-00884880

\section{HAL Id: hal-00884880 \\ https://hal.science/hal-00884880}

Submitted on 1 Jan 1986

HAL is a multi-disciplinary open access archive for the deposit and dissemination of scientific research documents, whether they are published or not. The documents may come from teaching and research institutions in France or abroad, or from public or private research centers.
L'archive ouverte pluridisciplinaire HAL, est destinée au dépôt et à la diffusion de documents scientifiques de niveau recherche, publiés ou non, émanant des établissements d'enseignement et de recherche français ou étrangers, des laboratoires publics ou privés. 


\section{Obtention de la forme sexuée de Rhizoctonia solani Kühn : Thanatephorus cucumeris (Frank) Donk}

Pierre CAMPOROTA

I.N.R.A., Station de Recherches sur la Flore pathogène dans le Sol, 17, rue Sully, F 21034 Dijon Cedex

RÉSUMÉ

Le but de cette brève note est de signaler l'obtention de la forme sexuée de Rhizoctonia solani (Thanatephorus cucumeris) au moyen de deux techniques différentes. Kühn).

The purpose of this short note is to report the production of basidia in Thanatephorus cucumeris by two different techniques.

\section{INTRODUCTION}

Le genre Rhizoctonia, champignon phytopathogène du sol, regroupe sous un même aspect morphologique la forme stérile de deux basidiomycètes : Thanatephorus cucumeris (Frank) Donk et Ceratobasidium (Bourd.) Rogers.

L'appartenance de souches de Rhizoctonia à l'un ou l'autre genre peut être déterminée en induisant le développement de la phase sexuée ou en observant le nombre de noyaux par article mycélien. PARMETER et al. (1967) ont montré en effet que les souches ayant pour forme sexuée Thanatephorus cucumeris possèdent plus de 2 noyaux par article (en moyenne 8) et correspondent à l'espèce Rhizoctonia solani Kühn ; les souches appartenant au genre Ceratobasidium ont des articles constamment binucléés et se rapportent aux espèces : Rhizoctonia cerealis Van der Hoeven, $R$. fragariae Hussain \& Mc Keen, $R$. fraxini Cast., $R$. callae Cast.

Les procédés de coloration nucléaire (TU \& KIMBROUGH, 1973 ; BURPEE et al., 1978 ; HERR, 1979) sont d'une mise en œuvre simple et rapide ; par contre, l'induction de la forme sexuée implique la mấtrise de techniques plus complexes.

Il existe 2 procédés pour induire la forme sexuée : la $1^{\text {re }}$ consiste à recouvrir une culture de la souche sur milieu gélosé par une couche de terre désinfectée (STRETTON et al., 1964 ; TU \& KIMBROUGH, 1975) ; la $2^{\mathrm{e}}$ consiste à cultiver le champignon d'abord sur un milieu gélosé riche, puis à le transférer sur un milieu gélosé pauvre (WHITNEY \& PARMETER, 1963 ; MURRAY, $1982 a$ et $b$; ADAMS \& BUTLER, $1983 a$ et $b$ ).

Cette note a pour but de rapporter l'obtention de la forme sexuée de $R$. solani en utilisant ces 2 procédés.

\section{MATÉRIEL ET MÉTHODE}

\section{A. Technique de la couche de terre}

L'essai a été réalisé en utilisant une souche isolée dans un sol maraîcher de la région d'Avignon. Son appartenance à l'espèce $R$. solani a été préalablement confirmée par le dénombrement des noyaux présents dans un article mycélien : une goutte de colorant (bleu d'aniline 1 p. 100 dans de l'alcool à 2 p. 100) a été déposée à la frange d'une culture sur milieu malt âgée de $48 \mathrm{~h}$, elle a été recouverte d'une lamelle de microscope ; l'observation a été faite $2 \mathrm{~h}$ après au grossissement $400 \mathrm{X}$.

Des boîtes de Petri de $60 \mathrm{~mm}$ de diamètre et contenant $8 \mathrm{ml}$ de milieu PDA sont ensemencées par un 
fragment prélevé à la frange d'une culture de la souche considérée. On laisse la colonie se développer pendant $13 \mathrm{j}$ à $25^{\circ} \mathrm{C}$ à l'obscurité puis on dépose une couche de $1 \mathrm{~cm}$ du mélange terreux couramment utilisé dans nos serres, désinfecté le jour même à $70^{\circ} \mathrm{C}$ par mélange air-vapeur pendant $30 \mathrm{mn}$. La terre est amenée à 60 p. 100 de sa capacité de rétention au moyen d'eau stérile, les boîtes sont fermées et placées pendant $5 \mathrm{j}$ à $25^{\circ} \mathrm{C}$ sous un éclairage d'intensité 1500 lux environ et une photopériode de $12 \mathrm{~h}$. Ce temps écoulé, les boîtes sont ouvertes et demeurent dans les mêmes conditions d'environnement ; l'humidité de la couche de terre est réajustée par pesée une fois par jour avec de l'eau stérile.

Au bout de 10 à $11 \mathrm{j}$, la terre se couvre d'un feutrage gris réparti irrégulièrement : l'observation à la loupe binoculaire $(G \times 100)$ montre qu'il est formé par des touffes fertiles composées de basides à tous les stades de maturité : probaside, croissance des stérigmates (en moyenne 4) et émission de basidiospores qui se poursuit pendant environ $8 \mathrm{j}$ (fig. 1).

\section{B. Technique du transfert de milieu}

Pour cet essai, la souche de $R$. solani utilisée nous a été fournie par A. OGoshi (Université de Sapporo, Japon) ; c'est une souche de référence pour le groupe d'anastomose 1 .

La culture sur milieu riche est faite sur du malt gélosé (cristomalt à $2 \mathrm{p}$. 100) pendant $8 \mathrm{j}$ à $25^{\circ} \mathrm{C}$ et à l'obscurité. Ensuite, un explantat de la colonie est déposé sur un milieu eau gélosée à 1,5 p. 100. Les boîtes de Petri, ainsi ensemencées, sont soumises aux mêmes conditions d'environnement pendant 5 à $6 \mathrm{j}$ au terme desquels on voit se former, à la périphérie de la colonie, des amas jaunâtres correspondant à des touffes fertiles (fig. 2).

\section{DISCUSSION}

Bien plus qu'un moyen d'identification des souches de Rhizoctonia, la possibilité d'obtenir la forme parfaite $T$. cucumeris ouvre une voie fort intéressante pour des études génétiques.

$R$. solani est un champignon à croissance rapide et simple à manipuler du fait de la taille de ses hyphes; il représente un modèle bien adapté pour progresser dans la mise en évidence des composantes du pouvoir pathogène et, par là, dans l'amélioration des moyens de lutte.

Il faut toutefois préciser que la forme sexuée de $R$. solani a été obtenue pour 2 souches seulement. Il convient maintenant de standardiser les conditions d'expérimentation de façon à obtenir une technique reproductible. A notre avis, ceci ne pourra valablement se faire qu'avec le $2^{\mathrm{e}}$ procédé du transfert de milieu, car la complexité du milieu sol rend très aléatoire la normalisation du $1^{\text {er }}$ procédé d'obtention du stade sexué de $R$. solani.

Reçu le 2 août 1985 . Accepté le 19 novembre 1985.

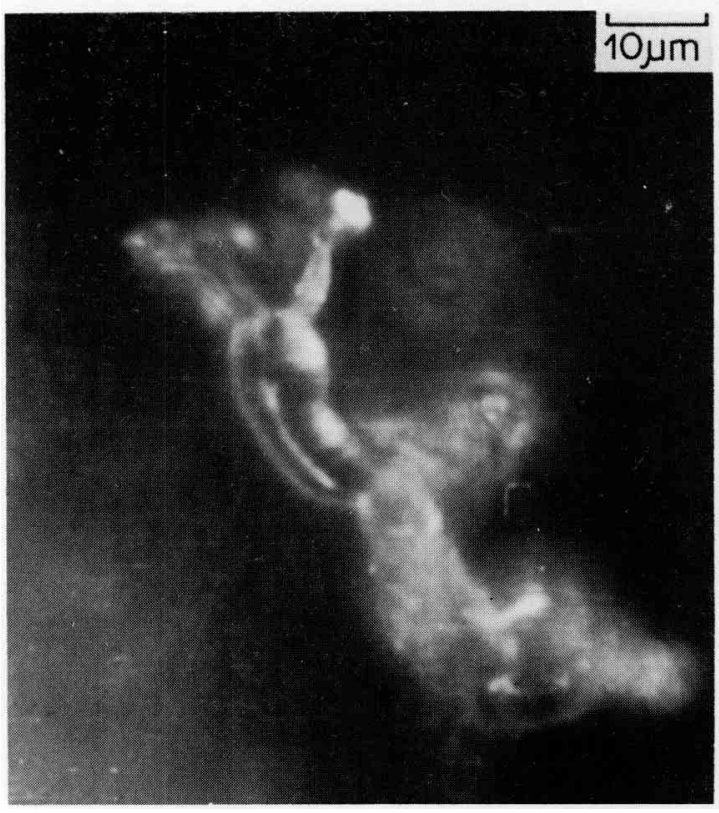

Figure 1

Baside mûre de Thanatephorus cucumeris observée in situ (Dispositif microscopique Ultropac-Zeiss), montrant stérigmates et spores. (L_ : $10 \mu \mathrm{m}$ ).

Mature basidium of $\mathrm{T}$. cucumeris observed in situ (Apparatus Ultropac-Zeiss), showing sterigmata and spores.

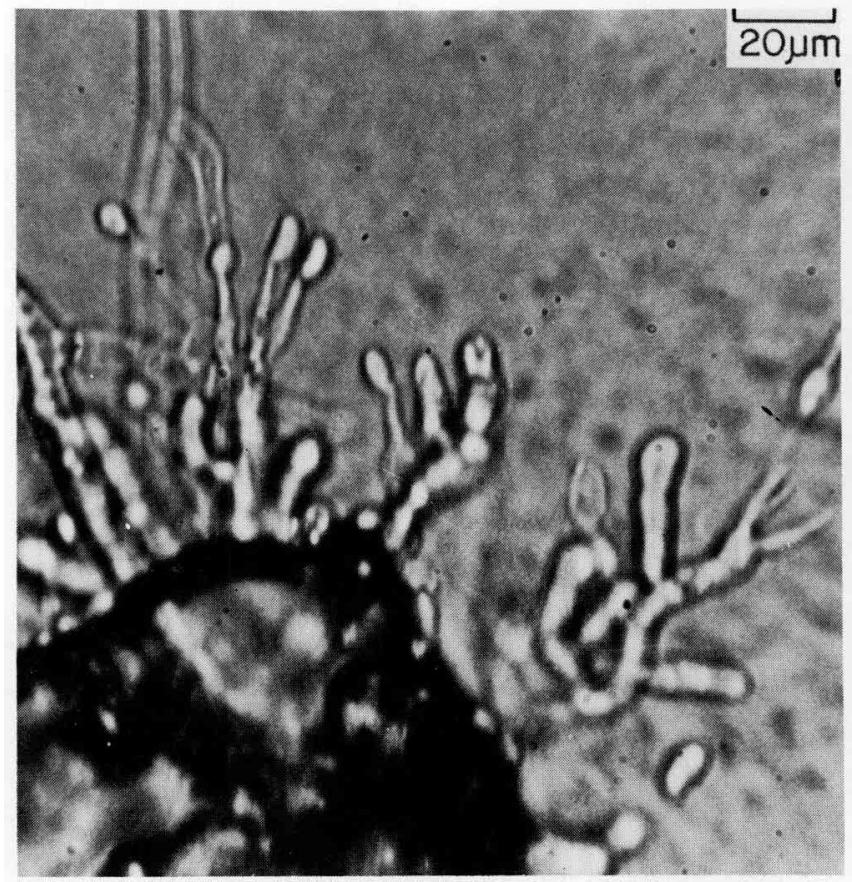

Figure 2

Détail de la touffe fertile. De droite à gauche, on voit une baside n'ayant pas germé, une probaside, puis une baside mûre portant 3 stérigmates et spores. (_ـ $20 \mu \mathrm{m})$.

Detail of the hymenium. From right to left : a non-germinated basidium, a probasidium, then a mature basidium with 3 sterigmata and spores. 


\section{RÉFERENCES BIBLIOGRAPHIQUES}

Adams G. C., Butler E. E., 1983a. Influence of nutrition on the formation of basidia and basidiospores in Thanatephorus cucumeris. Phytopathology, 73 (2), 147-151.

Adams G. C., Butler E. E., 1983b. Environmental factors influencing the formation of basidia and basidiospores in Thanatephorus cucumeris. Phytopathology, 73 (2), 152-155.

Burpee L. L., Sanders P. L., Cole H. Jr., Kim S. H., 1978. A staining technique for nuclei of Rhizoctonia solani and related fungi. Mycologia, 70, (6), 1281-1283.

Herr L. J., 1979. Practical nuclear staining procedures for Rhizoctonia-like fungi. Phytopathology, 69 (9), 958-961.

Murray D. I. L., 1982a. Cultural conditions influencing basidium formation in the Ceratobasidiaceae. Aust. J. Bot., 32, 101-108.

Murray D. I. L., 1982b. A modified procedure for fruiting Rhizoctonia solani on agar. Trans. Br. Mycol. Soc., 79 (1), 129-135.
Parmeter J. R. Jr., Whitney H. S., Platt W. D., 1967. Affinities of some Rhizoctonia species that resemble mycelium of Thanatephorus cucumeris. Phytopathology, 57 (2), 218-223.

Stretton H. M., Mc Kenzie A. R., Baker K. F., Flentje N. T., 1964. Formation of the basidial stage of some isolates of Rhizoctonia. Phytopathology, 54 (9), 1093-1095.

Tu C. C., Kimbrough W. J., 1973. A rapid staining technique for Rhizoctonia solani and related fungi. Mycologia, 65, 941-944.

Tu C. C., Kimbrough W. J., 1975. A modified soil over culture method for inducing basidia in Thanatephorus cucumeris. Phytopathology, 65 (6), 730-731.

Whitney H. S., Parmeter J. R. Jr., 1963. Synthesis of heterocaryons in Rhizoctonia solani Kühn. Can. J. Bot., 41, 879-886. 